\title{
DRIVER REHABILITATION IN PARKINSON'S DISEASE USING A DRIVING SIMULATOR: A PILOT STUDY
}

\author{
Ergun Uc ${ }^{1,5}$, Matthew Rizzo ${ }^{1,2,3}$, Steven Anderson ${ }^{2}$, Jessica Lawrence ${ }^{1}$, \& Jeffrey Dawson ${ }^{4}$ \\ ${ }^{1}$ Department of Neurology, Carver College of Medicine, University of Iowa \\ ${ }^{2}$ Department of Biostatistics, College of Public Health, University of Iowa \\ ${ }^{3}$ Department of Mechanical and Industrial Engineering, University of Iowa \\ ${ }^{4}$ Public Policy Center, University of Iowa \\ ${ }^{5}$ Neurology Service, Veterans Affairs Medical Center of Iowa City \\ Iowa City, Iowa, USA \\ Email: ergun-uc@uiowa.edu
}

\begin{abstract}
Summary: Parkinson's disease (PD) impairs driving performance. In this pilot study, four drivers with PD (selected based on poor road driving performance in the past) participated in a rehabilitation program using a driving simulator. Two different training drives (\#1- multiple intersections of varying visibility and traffic load, where an incurring vehicle posed a crash risk, \#2- various scenarios on decision making, hazard perception and response) were administered in each session (total 3 sessions once every 1-2 weeks) with immediate feedback after the drives. We observed reduction in crashes in drive \#1 and improved scores on drive \#2 in the simulator. In addition, 3 subjects showed marked improvements in their total error counts on a standard road test between baseline and post-training sessions, one subject stayed stable. These findings suggest that our simulator training program is feasible and potentially useful in impaired drivers with PD.
\end{abstract}

\section{INTRODUCTION}

Patients with Parkinson's disease (PD) have cognitive (attention, executive, visuospatial, memory), visual, and motor impairments and have shown poorer performance on road driving tests [Wood et al. 2005; Uc et al. 2009b; Devos et al. 2007] and driving simulator experiments [Stolwyk et al. 2005; Uc et al. 2009a] compared to neurologically normal drivers. Additional challenges such as driving under low visibility conditions [Uc et al. 2009a], route finding [Uc et al. 2007], search for landmarks, [Uc et al. 2006a], or auditory verbal distractions [Stolwyk et al. 2006; Uc et al. 2006b] can deteriorate their driving ability further. Drivers with PD are at higher risk for driving cessation [Uc et al. 2010]. Despite the increased safety risk in drivers with PD, there are no established programs to improve driving in PD. In a pilot study using repetitive intersection with crash risk in a simulator, we had shown before that crash rates and reaction times of drivers with PD can be improved on the same platform (simulator) [Dawson et al. 2009].

The goal of this pilot study was to determine if the gains in the simulator in drivers with PD transferred to improved driving performance on the road. The intervention involved repeated presentation of driving scenarios aimed at enhancing procedural memory, and training on visual perception, decision making, attention, and speed skills, coupled with individually-tailored feedback after each drive. We tested the road safety performance at baseline and after administering this simulator training program. 


\section{METHODS}

\section{Subjects}

Drivers from a recently completed cohort study [Uc et al. 2009b] who were still continuing to drive were consecutively invited in order of decreasing total error counts in their last drive. Four drivers with PD were enrolled. All these 4 subjects were from outside of Iowa City (i.e., nonlocal residents), but and had driven the same route in the same instrumented vehicle within 2-3 years before participation in this pilot study.

\section{Road test}

These subjects underwent a baseline road test (of 30 minutes) [Uc et al. 2009b] immediately before training and just after the last training session except one subject (\#2) who underwent the post-training drive with a 2 week delay due to weather conditions. The post-training drive was given in the reverse direction of the baseline drive. The video records of the drives were reviewed and scored per Iowa DOT error classification by a certified driving instructor, who was blinded to the status of the drives (baseline vs. post-training) [Uc et al. 2009b]. Error counts (total, "serious", on-task, no-task) were tabulated for each subject for baseline and post-training drives. The "serious" errors were those that were The "serious" errors were those that were judged as having the potential of leading to a crash or injury [Uc et al. 2009b]. On-task errors referred to errors during secondary tasks, e.g., route finding, or auditory verbal distractions route following [Uc et al. 2007], search for landmarks,[Uc et al. 2006a], or Paced Serial Addition Task (PASAT) [Uc et al. 2006b].

\section{Driving Simulator}

Our driving simulator, SIREN [Dawson et al. 2009; Uc et al. 2009a], comprises a 1994 GM Saturn, embedded electronic sensors, miniature video cameras for recording driver performance, a sound system and surrounding screens $\left(150^{\circ}\right.$ forward FOV, $50^{\circ}$ rear FOV), four LCD projectors with image generators, an integrated host computer, and another computer for scenario design, control, and data collection. A tile-based scenario development tool (DriveSafety, Salt Lake City, UT) was used.

\section{Simulator Training}

Three simulator training sessions included two drives during each session, and were administered in 1-2 week intervals. Immediate standardized, but individually-tailored, feedback was given after each drive based on prespecified triggers (below).

Drive \#1. In each training session the driver passed through 20 intersections on a simulated twolane highway, one mile apart, from each other, with waiting vehicles positioned in one crossing lane and in the opposing lane. The level of difficulty of the intersection was increased gradually by shortening the TTI trigger (the longer the TTI, the easier to avoid collision), adding oncoming traffic to the opposite lane, and changing the visibility (daylight vs. fog), as depicted in Table 1 of [Dawson et al. 2009]. The driver was advised to drive close to the speed limit and a honking 
sound alerted the driver if speed fell below 50 mph (except within 10 seconds of an intersection). Some intersections were "inactive" without any incursion occurring. In "active" intersections, the vehicle on the right pulled out in front of the driver, triggered in response to driver speed by a predetermined time-to-intersection (TTI). This event required immediate decision making and action by the subject to attempt to avert a crash. Optimal response involved releasing the accelerator, applying the brake, and making steering corrections as needed to remain within the lane. The outcome measure was number of crashes in each drive. We tabulated the number of crashes across visits.

Drive \#2. This was a 15-20 minute drive on a 2-lane, 55 mph rural highway. During each visit the order of scenarios were different to diminish learning effects. Following scenarios were used:

1. Rear-end collision avoidance at intersections

- Description: The driver approached an intersection and became trapped behind a lead vehicle that remained inexplicably stopped at a green traffic light and proceeded forward only after the light turned red. There was a potential for a rear-end collision.

- Feedback triggers: Collision or an unsafe maneuver (e.g., steering left into oncoming traffic), following the lead vehicle into the intersection in green light.

2. Response to an emergency vehicle

- Description: In this scenario, drivers encountered a police car with flashing lights on the shoulder of the road with no oncoming traffic while driving on $55 \mathrm{mph}$ rural 2-lane highway.

- Triggers for feedback: Failure to select appropriate behavioral response (changing lane or gradually slowing down using appropriate head and mirror checks), creating unsafe situations such as abrupt decelerations, stopping in the middle of the road.

3. Response under stress: Animal encounter while being tailgated

- Description: In this scenario, the driver was tailgated by an aggressive, honking SUV driver. Within one second of the honk, a dog darts to the road from the right.

- Triggers for feedback: Sudden stopping or slowing down in the lane while if the SUV behind the driver is in striking distance. Failure to check the rear view mirror when SUV honks.

4. Window Rolling Task (WRT): Motor multitasking

- Description: WRT was administered in a segment with intermittent oncoming traffic. The driver was asked to roll the window all the way down and then up.

- Feedback triggers: Crash with oncoming traffic; unsafe driving behavior such as lane deviations, erratic steering, or driving more than 10 miles above or below the speed limit; not ceasing engagement in WRT despite encountering increased risks for collision due to increased oncoming traffic or while having erratic steering and lane deviations.

5. Intersection incursion scenario

- Each drive ended with this event, which was similar to the intersection incursions in Drive \#1 (TTI=4.0 sec).

Each scenario was scored in a semiquantitative manner over a 0 - 2 scale: $0=$ poor performance, $1=$ suboptimal (acceptable but with errors), 2=optimal. The criteria for these scores in each scenario are given in Table 2 . The total score the drive was obtained by adding up all scenario scores ranged 0-10. We tabulated the score for Drive \#2 across visits. 


\section{RESULTS}

Our subjects had mild-moderate PD with decreased visual contrast sensitivity. Table 1 shows the demographic data, and their cognitive, visual, and motor performance.

Table 1. The characteristics of the PD subjects

\begin{tabular}{cccccccc}
\hline ID & Age & Sex & MMSE & Hoehn-Yahr stage & CS & Motor UPDRS & ADL UPDRS \\
\hline 1 & 70 & Male & 28 & 2.5 & 1.65 & 18 & 6 \\
2 & 65 & Male & 29 & 2.5 & 1.35 & 18 & 11 \\
3 & 70 & Male & 24 & 3 & 1.35 & 25 & 17 \\
4 & 71 & Male & 28 & 2.5 & 1.35 & 26 & 11 \\
\hline
\end{tabular}

MMSE=Mini-Mental Status Exam, CS=contrast sensitivity, ADL= activities of daily living, UPDRS=Unified

Parkinson's Disease Rating Scale

Table 2. Performance in serial simulator training sessions

\begin{tabular}{|c|c|c|c|c|}
\hline \multicolumn{4}{|c|}{ Simulator Training Sessions } & \multirow{2}{*}{$\begin{array}{c}\text { Tasks and Scoring } \\
\underline{\text { DRIVE } 1}\end{array}$} \\
\hline ID & I & II & III & \\
\hline 1 & 0 & 0 & 0 & Repeated intersections \\
\hline 2 & 3 & 1 & 1 & Score indicates \# of crashes that occurred during this drive, which consisted of 20 \\
\hline 3 & 6 & 1 & 2 & intersections, each 1 mile apart. In 11 of 20 intersections, a vehicle illegally incurred into the \\
\hline 4 & 3 & 1 & 0 & intersection causing a collision risk. \\
\hline ID & I & II & III & DRIVE 2 \\
\hline 1 & 1 & 1 & 1 & Emergency Vehicle \\
\hline 2 & 1 & 1 & 2 & $0=$ No reaction, no merge, no reduction in speed \\
\hline 3 & missing* & 0 & 1 & 1 = Slowing down or merging into other lane without head checks \\
\hline 4 & 1 & 1 & 2 & 2 = Merged into other lane, used turn signal with head check \\
\hline 1 & 2 & 2 & 1 & Go/No Go at the Traffic Signal \\
\hline 2 & 1 & 1 & 2 & $0=$ Hits lead vehicle, deviates left or right, enters intersection through red \\
\hline 3 & missing & 0 & 1 & 1 = Stop too far away, stop abruptly \\
\hline 4 & 0 & 1 & 1 & 2 = Stops before hitting lead vehicle, doesn't enter intersection on red \\
\hline 1 & 1 & 1 & 2 & Window Rolling \\
\hline 2 & 0 & 0 & 1 & $0=$ Can't do task, large lane deviations into other lane ( $>1 / 2$ of car in other lane) \\
\hline 3 & missing & 0 & 0 & $1=$ Increased swerving in lane, minor lane deviations $(<1 / 2$ of car in other lane) \\
\hline 4 & 2 & 1 & 1 & 2 = Completes task, no lane deviations \\
\hline 1 & 0 & 0 & 0 & SUV-Dog \\
\hline 2 & 2 & 2 & 2 & $0=$ No reaction to honk, rear-end collision w/ SUV, stopping, deviating left or right \\
\hline 3 & missing & 0 & 1 & 1 = Hits dog but slows down, no reaction to honk \\
\hline 4 & 1 & 2 & 2 & 2 = Reaction to honk, hit dog without slowing down, no rear-end collision w/ SUV \\
\hline 1 & missing & 2 & 2 & Intersection Incursion \\
\hline 2 & 2 & 0 & 2 & $0=$ Crash \\
\hline 3 & missing & 0 & 1 & 1 = Risky avoidance \\
\hline 4 & 1 & 1 & 2 & $2=$ No crash \\
\hline 1 & 4 & 6 & 6 & Total Score $(0-10)$ \\
\hline 2 & 6 & 4 & 9 & A subject who completed all 5 scenarios in the drive perfectly would receive maximum 10 \\
\hline 3 & missing & 0 & 4 & points. Subjects are assigned missing value if they cannot attempt a scenario or the whole \\
\hline 4 & 5 & 6 & 8 & drive due to simulator sickness. \\
\hline
\end{tabular}


Our drivers generally showed improvement in simulator tasks across visits. (Table 2). The number of collisions in Drive 1 and the total score in Drive 2 improved in all subjects from the first to last simulator session.

Three of 4 subjects showed improvement in total road error counts (19\%, 42,\%, 54\%), and the subject with worst cognitive status and parkinsonism (PDI3) stayed essentially stable (Table 3). The mean (SD) improvement was 13.0 (12.2). There was substantial improvement (35\%, 35\%, 45\%, 50\%) in the "on task" error counts which were observed during multitasking in tasks like navigation [Uc et al. 2007] and visual search [Uc et al. 2006a]. Of note, the subject with worst MMSE score and worst total error count at baseline showed 45\% improvement with on-task errors There was also consistent improvement across all subjects in intersection (traffic signal and stop sign) related errors.

Table 3. Road safety performance before and after training

\begin{tabular}{ccccc}
\hline ID & Baseline & Post-training & Difference & ROAD TEST OUTCOMES \\
\hline 1 & 48 & 28 & $-20(-42 \%)$ & Total Error count: All at-fault safety errors \\
2 & 48 & 22 & $-26(-54 \%)$ & during the drive \\
3 & 55 & 56 & $1(2 \%)$ & \\
4 & 37 & 30 & $-7(-19 \%)$ & \\
1 & 17 & 11 & $-6(-35 \%)$ & On Task Error count: All at-fault safety errors during \\
2 & 17 & 11 & $-6(-35 \%)$ & the drive that were committed while multitasking \\
3 & 31 & 17 & $-14(-45 \%)$ & such as navigating a newly learned route or visual \\
4 & 16 & 8 & $-8(-50 \%)$ & search of landmarks \\
1 & 2 & 2 & 0 & "Serious” Errors: Judged as having the potential of \\
2 & 6 & 1 & -1 & leading to a crash or injury \\
3 & 1 & 1 & 0 & -3 \\
4 & 4 & 1 & & \\
\hline
\end{tabular}

\section{DISCUSSION}

To our knowledge, there are no published reports on driver rehabilitation in PD except an earlier pilot study by our group showing feasibility of simulator training [Dawson et al. 2009]. The literature on driving rehabilitation of elderly or neurologically impaired drivers is also limited. A systematic review of randomized controlled studies on older driver retraining [Kua et al. 2007] revealed limited evidence that physical retraining and visual perception training [Roenker et al. 2003] improved driving related skills in older drivers. Educational interventions [Bedard et al. 2004; Owsley et al. 2004] showed moderate evidence that educational interventions improve driving awareness and driving behavior, but do not reduce crashes in older drivers. Short term trials using physical conditioning [Marottoli et al. 2007a] or classroom and road retraining [Marottoli et al. 2007b] showed improvements in post-training road test performances. An intense simulator training program (15 sessions over several weeks) led to significant improvements within the simulator and was associated with passing an official driving assessment [Akinwuntan et al. 2005]. However, there was no difference in driving cessation between the simulator training and control groups at 5 years [Devos et al. 2009]. 
There is evidence that cognitive rehabilitation can improve executive functions in PD [Sammer et al. 2006] suggesting that PD patients can be trainable using a driving simulator. Both our previous and the current pilot study showed the feasibility of simulator training intervention in PD with suggestions of potential efficacy. Our approach is based on observations in our large PD driver cohort and is aimed at improving visual perception, decision making, attention, speed, and procedural memory using scenarios stressing these abilities. We tried to consolidate training gains by providing immediate feedback. Our sample size does not allow us to make meaningful statistical inferences. However, the substantial and consistent improvement in the road error counts may suggest efficacy of the intervention in very short term. While transfer of improvements in simulator driving to the road could be one of the possible explanations of the road safety improvement, a short term practice effect on the route (despite using reverse direction) and tasks can partly explain the observed improvements. Based on this experience, we are conducting a preliminary driver rehabilitation project in PD where we are testing the feasibility and effect size of similar training scenarios at different durations and difficulty levels over a longer observation period.

Open questions in driving simulation training for elderly and neurologically impaired drivers include finding best target population, choice of scenarios, frequency and duration of training, need to combine with other rehabilitation modalities, measuring transfer effects from simulator to real life/road performance, and need for booster training to maintain potential benefit.

\section{ACKNOWLEDGEMENTS}

NIH/National Institute of Neurological Disorders and Stroke R01 NS044930 (Predicting Driver Safety in Parkinson's Disease) [EYU]; National Institute on Aging R01 AG17717 and R01 AG15071 [MR].

\section{REFERENCES}

Akinwuntan AE, De Weerdt W, Feys $\mathrm{H}$ et al. Effect of simulator training on driving after stroke: a randomized controlled trial. Neurology 2005; 65: 843-850.

Bedard M, Isherwood I, Moore E, Gibbons C, Lindstrom W. Evaluation of a re-training program for older drivers. Can J Public Health 2004; 95: 295-298.

Dawson JD, Rizzo M, Anderson SW, Dastrup E, Uc EY. Collision Avoidance Training Using a Driving Simulator in Drivers with Parkinson's Disease: A Pilot Study. In: Boyle LN, Lee JD, McGehee DV, Rizzo M, editors. Proceedings of Driving Assessment 2009: The Fifth International Driving Symposium on Human Factors in Driver Assessment, Training and Vehicle Design. Iowa City, Iowa: The University of Iowa; 2009. p. 154-60.

Devos H, Akinwuntan AE, Nieuwboer A et al. Comparison of the effect of two driving retraining programs on on-road performance after stroke. Neurorehabil Neural Repair 2009; 23: 699-705.

Devos H, Vandenberghe W, Nieuwboer A, Tant M, Baten G, De Weerdt W. Predictors of fitness to drive in people with Parkinson disease. Neurology 2007; 69: 1434-1441. 
Kua A, Korner-Bitensky N, Desrosiers J, Man-Son-Hing M, Marshall S. Older driver retraining: a systematic review of evidence of effectiveness. J Safety Res 2007; 38: 81-90.

Marottoli RA, Allore $\mathrm{H}$, Araujo KL et al. A randomized trial of a physical conditioning program to enhance the driving performance of older persons. J Gen Intern Med 2007a; 22: 590-597.

Marottoli RA, Ness PH, Araujo KL et al. A randomized trial of an education program to enhance older driver performance. J Gerontol A Biol Sci Med Sci 2007b; 62: 1113-1119.

Owsley C, McGwin G, Jr., Phillips JM, McNeal SF, Stalvey BT. Impact of an educational program on the safety of high-risk, visually impaired, older drivers. Am J Prev Med 2004; 26: 222-229.

Roenker DL, Cissell GM, Ball KK, Wadley VG, Edwards JD. Speed-of-processing and driving simulator training result in improved driving performance. Hum Factors 2003; 45: 218-233.

Sammer G, Reuter I, Hullmann K, Kaps M, Vaitl D. Training of executive functions in Parkinson's disease. J Neurol Sci 2006.

Stolwyk RJ, Triggs TJ, Charlton JL, Iansek R, Bradshaw JL. Impact of internal versus external cueing on driving performance in people with Parkinson's disease. Mov Disord 2005; 20: 846-857.

Stolwyk RJ, Triggs TJ, Charlton JL, Moss S, Iansek R, Bradshaw JL. Effect of a concurrent task on driving performance in people with Parkinson's disease. Mov Disord 2006; 21: 20962100.

Uc EY, Rizzo M, Anderson SW, Dastrup E, Sparks JD, Dawson JD. Driving under low-contrast visibility conditions in Parkinson disease. Neurology 2009a; 73: 1103-1110.

Uc EY, Rizzo M, Anderson SW, Sparks J, Rodnitzky RL, Dawson JD. Impaired visual search in drivers with Parkinson's disease. Ann Neurol 2006a; 60: 407-413.

Uc EY, Rizzo M, Anderson SW, Sparks JD, Rodnitzky RL, Dawson JD. Driving with distraction in Parkinson disease. Neurology 2006b; 67: 1774-1780.

Uc EY, Rizzo M, Anderson SW, Sparks JD, Rodnitzky RL, Dawson JD. Impaired navigation in drivers with Parkinson's disease. Brain 2007; 130: 2433-2440.

Uc EY, Rizzo M, Dastrup E, Liu D, Anderson SW, Dawson JD. Real-life driving outcomes in mild-moderate Parkinson's disease. Neurology 2010; 74(Suppl 2): A58.

Uc EY, Rizzo M, Johnson AM, Dastrup E, Anderson SW, Dawson JD. Road Safety in Drivers with Parkinson Disease. Neurology 2009b; 73: 2112-2119.

Wood JM, Worringham C, Kerr G, Mallon K, Silburn P. Quantitative assessment of driving performance in Parkinson's disease. J Neurol Neurosurg Psychiatry 2005; 76: 176-180. 